\title{
Articles
}

\section{Three generations of autoimmune diabetes: an extended family study}

\author{
I. F. Douek ${ }^{1}$, K. M. Gillespie ${ }^{1}$, R. J. Dix ${ }^{1}$, P. J. Bingley ${ }^{1}$, E. A. M. Gale ${ }^{1,2}$ \\ ${ }^{1}$ Diabetes and Metabolism, Division of Medicine, University of Bristol, Bristol, UK \\ ${ }^{2}$ Diabetes and Metabolism, Medical School Unit, Southmead Hospital, Bristol, UK
}

\section{Abstract}

Aims/hypothesis. One in four children with Type 1 diabetes in a population-based family study has an affected grandparent. We set out to study the clinical and immune features of diabetes in the grandparents' generation, and to examine sharing of HLA class II susceptibility haplotypes between grandparent and grandchild.

Methods. Of 5855 grandparents in the Bart's-Oxford family study, 428 (7.3\%) were known to have diabetes. Clinical data and samples were collected from 115 of 213 surviving affected grandparents and from 219 unaffected grandparents within the same families. Samples were tested for ICA and autoantibodies to GAD and IA-2, and typed for HLA-DRBI-DQA1$D Q B 1$. Transmission of HLA class II haplotype from affected and unaffected grandparents to the diabetic proband was compared.

Results. Of 115 affected grandparents studied, the median age at diagnosis was 61 years and at analysis was 73 years; $70 \%$ were diet or tablet treated and 30\% were on insulin. One or more islet autoantibodies were found in $26 \%$ and $66 \%$ had one or both of the high risk HLA class II susceptibility haplotypes $D R B 1 * 03-D Q A 1 * 0501-D Q B 1 * 0201$ or DRB1*04$D Q A 1 * 0301-D Q B 1 * 0302$. In 79 informative families the HLA class II haplotype of the affected grandparent was transmitted to the proband more frequently than expected overall $(59 \%, p=0.02)$, and in the insulintreated subgroups $(65 \%, p=0.03)$.

Conclusion/interpretation. A total of $7.3 \%$ of grandparents reported a clinical diagnosis of diabetes and $2.2 \%$ had features of Type 1 diabetes. Genetic susceptibility was shared between grandparents with diabetes and their affected grandchildren. Diabetes in the grandparents of children with Type 1 diabetes often has an autoimmune basis, even when it presents late in life and does not require insulin treatment. [Diabetologia (2003) 46:1313-1318]

Keywords Type 1 diabetes, Type 2 diabetes, classification, familial risk, islet autoantibodies, HLA.
Immune-mediated diabetes is not exclusively, or even typically, a disease of childhood. Presentation in later

Received: 5 February 2003 / Revised: 22 April 2003

Published online: 30 July 2003

C) Springer-Verlag 2003

Corresponding author: Prof. E. A. M. Gale, Diabetes and Metabolism, Medical School Unit, Southmead Hospital, Bristol, BS10 5NB, UK

E-mail: Edwin.Gale@bristol.ac.uk

Tel.: +44-117-9595337, Fax: +44-117-9595336

Abbreviations: BOX, Bart's-Oxford study of childhood diabetes; ICA, islet cell autoantibodies. life ranges across a spectrum; at one end are those who present acutely and require immediate insulin treatment, and at the other are those who present as Type 2 diabetes yet carry islet autoantibodies. Since the classification of immune-mediated diabetes was previously based solely upon an early requirement for insulin [1], the frequency of the condition could have been underestimated in older relatives of children with classic Type 1 diabetes. With this in mind, we examined the parents of children with Type 1 diabetes in an earlier study, and found that $3.1 \%$ had insulin-treated diabetes and $2.1 \%$ did not require insulin. When aetiological criteria were used, however, we found 
that $4.5 \%$ had immune-mediated diabetes and only $0.9 \%$ had a non-immune form of the disease; the latter estimate did not differ from reported rates in the background population [2].

A prolonged prodromal period with onset in later life is characteristic of many other autoimmune conditions, and it would not be surprising if a proportion of cases of Type 1 diabetes presented in this way; this may even have been the characteristic mode of onset in earlier generations. On this basis, we have speculated that a more permissive environment might lead to earlier clinical presentation in genetically predisposed subjects, and that earlier disease onset might partially explain the rising incidence of childhood onset Type 1 diabetes over the past 50 years [3,4]. Another example of earlier disease onset in response to a more permissive environment would be the current epidemic of obesityrelated childhood onset Type 2 diabetes [5]. We therefore aimed to establish whether examination of the grandparental generation would add biological support to this concept, and set out to determine the prevalence of immune-mediated diabetes in this generation, and to look for evidence of shared genetic susceptibility between affected grandparent and grandchild.

\section{Subjects and methods}

Grandparents with diabetes were identified through the families participating in the Bart's Oxford (BOX) study [6]. The BOX study is an ongoing prospective population based family study in the former Oxford Regional Health Authority area. Since it began in 1985, the study has recruited $88 \%$ of the families of children diagnosed with Type 1 diabetes below the age of 21. By the end of December 2001, 1693 families had been recruited into the study with $89 \%$ under regular follow up. A family history of diabetes in first and second degree relatives of the affected child was collected at study entry.

The diabetes status of the grandparents was updated annually by a questionnaire in all families under follow up. Families in which one or more grandparent was known to have diabetes were contacted to ascertain the health status of both affected and unaffected grandparents. They were also asked to provide contact details for all surviving grandparents if relatives thought this appropriate. Grandparents were sent an explanatory letter about the BOX study and were invited to join. Unaffected grandparents in these families were also recruited. A single visit was arranged at which detailed information about diagnosis of diabetes and time to insulin treatment were recorded. A medical history was obtained in all grandparents and included specific details about other autoimmune diseases. Grandparents were asked to provide blood samples for autoantibody assessment and genetic analysis, and those without diabetes were also invited to give a sample for $\mathrm{HbA}_{1 \mathrm{c}}$ analysis. Four individuals who did not wish to give blood were invited to provide a mouth swab for genotyping. Islet cell antibodies (ICA) and antibodies to glutamic acid decarboxylase (GAD) and the protein tyrosine phosphatase IA-2 were assessed in the remainder, and genotyping for $H L A-D R B 1, D Q A 1$ and $D Q B 1$ alleles was carried out in all cases.

General practitioners of unaffected grandparents who had an $\mathrm{HbA}_{1 \mathrm{c}}$ value above $6 \%$ were contacted and asked to carry out further screening for diabetes.

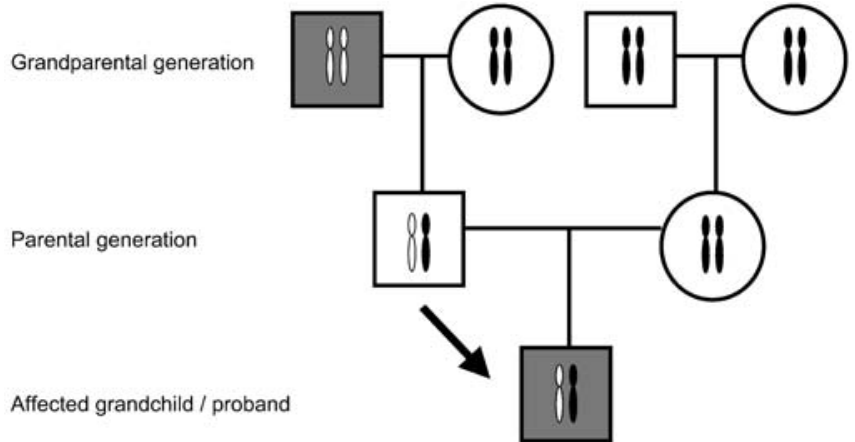

Fig. 1. Transmission of the HLA haplotype from affected grandparent through the parent to the proband. Grandparents or grandchildren affected with diabetes are shaded. The parental generation does not necessarily have diabetes but carries and transmits the disease-linked HLA haplotype to the next generation. The expected transmission of grandparental haplotypes from parent to proband is $50 \%$

Informed consent was obtained from all study subjects. The study was approved by the ethics committees of all centres involved in the study and was carried out in accordance with the Declaration of Helsinki as revised in 2000.

Assays. ICA were measured in sera by indirect immunofluorescence as described previously [7]. End point titres were converted to Juvenile Diabetes Foundation (JDF) units by comparison with a standard curve of $\log _{2}$ JDF units versus $\log _{2}$ of the end point titre of the standard sera. The threshold of ICA detection was $5 \mathrm{JDF}$ units. Antibodies to $\mathrm{GAD}_{65}$ and IA-2 ${ }_{\text {ic }}$ were measured by radioimmunoassay [7]. The ICA assay achieved $81 \%$ sensitivity with $86 \%$ specificity, the GAD antibody assay $91 \%$ sensitivity with $99 \%$ specificity, and the IA-2 antibody assay $74 \%$ sensitivity with $99 \%$ specificity in the first Immunology of Diabetes Society (IDS) combined antibody workshop [8].

HLA genotyping was carried out on DNA from blood or mouth swab samples. Details of DNA extraction methods and HLA class II analysis have been published [9]. Briefly, mouth swab extractions were carried out using a guanidium chloride/phenol:chloroform method. Low yield DNA samples from mouth swabs underwent whole genome amplification by primer extension preamplification where necessary. HLA analysis was carried out by polymerase chain reaction using sequence specific primers [10]. Samples were typed for $H L A-D R B 1 * 01-10$, for $D Q A 1 * 0201,0301,0302$ and 0501, and for subtypes of $D Q B 1 * 02-06$. Extended HLA genotypes were constructed. Subjects who did not have the high risk haplotypes $D R B 1 * 03$ $D Q A 1 * 0501-D Q B 1 * 0201$ or $D R B 1 * 04-D Q A 1 * 0301-D Q B 1 * 0302$ or the protective haplotype $D R B 1 * 02-D Q B 1 * 0602$ were reported as $D R X$.

$\mathrm{HbA}_{1 \mathrm{c}}$ was measured by high pressure liquid chromatography (HPLC) using a $\mathrm{HAH}_{140}$ analyser (BioMen Diagnostics, Falcon Business Park, Finchampstead, UK).

Data analysis. Classification of diabetes in affected grandparents was based on current treatment, time from diagnosis to insulin treatment and the presence or absence of one or more islet cell autoantibodies. Grandparents were classified as having Type 1 diabetes if permanent insulin therapy was started within 12 months of diagnosis and/or one or more islet-cell autoantibody was present. Affected grandparents were considered to have Type 2 diabetes if they did not require insulin and were antibody negative. 
Autoantibody analyses for ICA, GAD and IA-2 were considered positive when above the 97.5th centile of a control population of schoolchildren [7]. This corresponded to 5 JDF units for ICA, 1.6 units for GAD antibodies and 0.9 units for IA-2 antibodies.

HLA DRB1-DQA1-DQB1 genotype was analysed in the proband, parents and affected and unaffected grandparent to study transmission of all typed HLA haplotypes through the three generations. The frequency of transmission of the HLA haplotype from the affected grandparent to the proband was calculated and compared by Chi squared testing (Fig. 1). A $p$ value of less than 0.05 was considered significant.

\section{Results}

Grandparents known to have diabetes. One or more grandparent had a diagnosis of diabetes in 369 of 1500 families $(24.6 \%)$ under follow up. In total 428 $(7.3 \%)$ of 5855 grandparents were known to have diabetes, one grandparent was affected in 315 families, two grandparents in 49 families and three grandparents in five families. Of 213 surviving grandparents, 115 were willing and able to provide further information and samples (Fig. 2). The characteristics of the study group matched those of the whole group of grandparents with diabetes on whom treatment details were available (Table 1).

The median age of the affected group at the time of study was 73 years, range 46 to 89 years. Age at onset of diabetes ranged from 7 to 81 , median 61 years. The overall male to female ratio in the affected group was $1: 1.05,30 \%$ were treated with insulin and time to insulin from diagnosis varied from 0 to 25 years. The immunogenetic features of the subgroup studied are shown in Table 2. Autoantibody analysis was carried out in 111 affected grandparents, as four did not give a blood sample. One or more islet autoantibodies were present in $26 \%$ and $8 \%$ had two or more; $14 \%$ had ICA, median 10.5 JDF units, range 7 to over $80 ; 18 \%$ GAD antibodies, median value 17 units, range 2 to over 100; and 3\% IA-2 antibodies, median 3, range 1.5 to 3.2. Samples

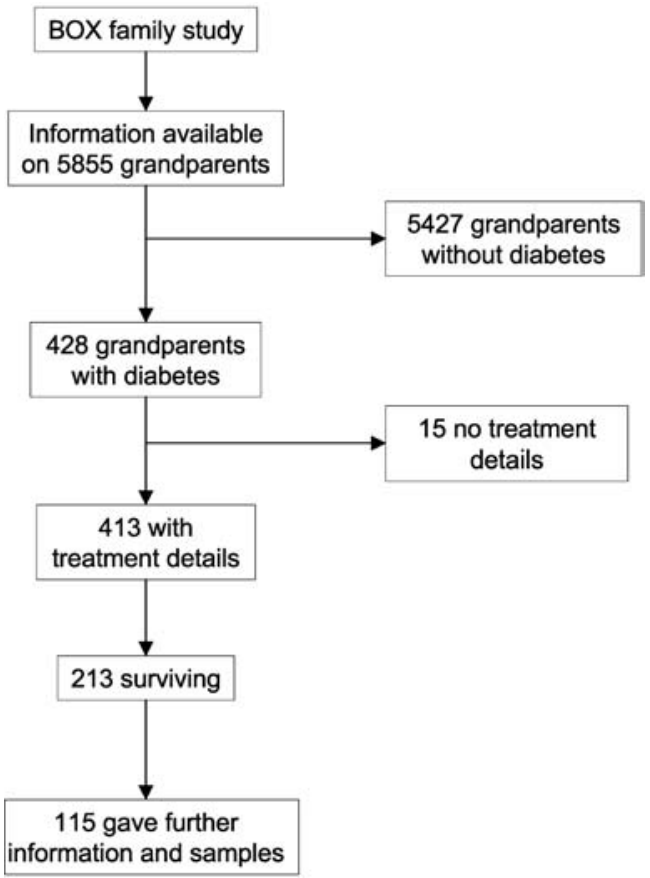

Fig. 2. Grandparents in the BOX study

were taken from 10 months to 48 years after diagnosis. The highest risk HLA genotype $D R B 1 * 03-D Q A 1 * 0501$ $D Q B 1 * 0201 / D R B 1 * 04-D Q A 1 * 0301-D Q B 1 * 0302$ was present in $9 \%$ of the 115 affected grandparents, $66 \%$ had either one or both of the above haplotypes and $15 \%$ had the protective HLA haplotype $D Q B 1 * 0602$.

Of the 115 affected grandparents assessed, 36 were classified as having Type 1 diabetes (Fig. 3). Seven of these had started insulin treatment within 12 months of diagnosis, 20 had one or more islet autoantibody, and 9 fulfilled both criteria. Median age at diagnosis in the Type 1 diabetes group was 58, range 7 to 77 years. In those who started insulin early this was 52 (range 7-75 years), and was 63 (range 52-77 years) in those with antibodies but not on insulin. There were 62 grandparents who were both antibody negative and not insulin-

Table 1. Characteristics of all grandparents with diabetes

\begin{tabular}{lcc}
\hline & Treatment details available & Subgroup tested \\
\hline Number of subjects & 413 & 115 \\
Sex (male:female) & $213: 200$ & $56: 59$ \\
paternal grandparent & $116: 97$ & $29: 16$ \\
maternal grandparent & $97: 103$ & $27: 43$ \\
Median age at diagnosis in years (range) & 61 (2-95) & $61(7-81)$ \\
Median current age in years (range) & 73 b (46-94) & $73(46-89)$ \\
Diet/tablet treated & $290(70 \%)$ & $80(70 \%)$ \\
Insulin & $123(30 \%)$ & $35(30 \%)$ \\
Parents with diabetes bridging affected grandparent and child & $38(9 \%)$ & $11(10 \%)$ \\
Fathers & 26 & 7 \\
Mothers & 12 & 4 \\
\hline
\end{tabular}

a Information available from 254

$\mathrm{b}$ Information from 188 surviving 


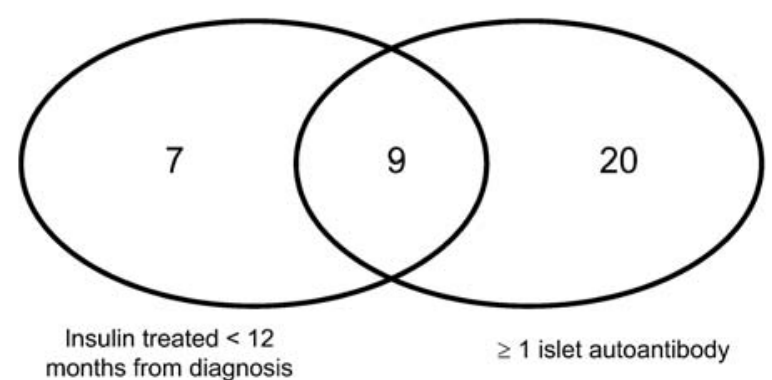

Fig. 3. Basis of classification of Type 1 diabetes in grandparents. Of the 115 grandparents studied, 79 had either Type 2 diabetes $(n=62)$ or were classified as indeterminate $(n=17)$, and 36 had Type 1 diabetes. Of these seven were insulin treated within 12 months of diagnosis, in 20 one or more islet autoantibody above the 97.5 th centile was present and both factors were found in nine

Table 2. Characteristics of grandparents with diabetes analysed $(n=115)$

Characteristic

(\% of those tested)

Current treatment

Insulin

$<12$ months after diagnosis

$\geq 12$ months after diagnosis

$16(14)$

$19(17)$

Tablets

$64(56)$

Diet

$16(14)$

Autoantibody status (111 tested)

No antibodies

GAD antibodies $\geq 97.5^{\text {th }}$ centile

ICA antibodies $\geq 97.5^{\text {th }}$ centile

IA 2 antibodies $\geq 97.5^{\text {th }}$ centile

$82(74)$

$20(18)$

$16(14)$

$3(3)$

$29(26)$

$9(8)$

HLA class II (115 tested)a

$\begin{array}{lc}D R 3 / D R 4 & 10(9) \\ D R 4 / D R 4 & 3(3) \\ D R 3 / D R 3 & 2(2) \\ D R 4 / D R X & 27(23) \\ D R 3 / D R X & 26(23) \\ D R 4 \text { or } D R 3 / D R B 1 * 02-D Q B 1 * 0602 & 9(8) \\ D R B 1 * 02-D Q B 1 * 0602 / X & 8(7) \\ \text { None of the above } & 30(26)\end{array}$

a Haplotype codes are:

$D R 3$ indicates the extended haplotype $D R B 1 * 03-D Q A 1 * 0501$ DQB1-0201

$D R 4$ indicates the extended haplotype $D R B 1 * 04-D Q A 1 * 0301$ $D Q B 1-0302$

$D R X$ indicates any other extended haplotype from those shown treated, and these were considered to have Type 2 diabetes. Median age at diagnosis in this group was 68, range 32 to 81 years. No classification was made in 17, including 4 who had not provided blood samples for antibody estimation, and 13 who started insulin more than 12 months after diagnosis and were antibody negative 2 to 35 years after disease onset. The highest risk HLA genotype $D R B 1 * 03-D Q A 1 * 0501-D Q B 1 * 0201 /$ $D R B 1 * 04-D Q A 1 * 0301-D Q B 1 * 0302$ was present in $17 \%$ of the 36 grandparents with Type 1 diabetes and $5 \%$ of the 62 with Type 2 diabetes; $66 \%$ and $69 \%$ respectively had either one or both of the above haplotypes and $6 \%$ and $18 \%$ respectively had the protective HLA haplotype $D Q B 1 * 0602$.

We were able to extrapolate our findings from the subgroup analysed to the grandparents as a whole, since the baseline features were similar (Table 1). Assessment of the 115 grandparents led to the classification of $36(31.3 \%)$ as having Type 1 diabetes. The number of grandparents with Type 1 diabetes from the group as a whole can then be estimated as 129 , that is $31.3 \%$ of the 413 grandparents with treatment details. Thus 129 of 5855 or $2.2 \%$ of grandparents of a child with Type 1 diabetes could be estimated as having Type 1 diabetes.

Grandparents not known to have diabetes. Information and blood or mouth swab samples were provided by 219 grandparents not known to have diabetes. Median age at study entry was 71 years, range 47 to 93 . One or more islet antibodies were present in $15 \%$ of the 211 blood samples collected and two or more in 2\%; $10 \%$ ICA, median 10 JDF units, range 7 to over 80 ; $6 \%$ had GAD antibodies, median value 5.2 units, range 1.6 to 96 ; and $1.4 \%$ IA-2 antibodies, median 1.2 units, range 1.0 to 1.8 . $\mathrm{HbA}_{1 \mathrm{c}}$ was above $7 \%$ in $3 \%$ of the samples measured, one antibody was increased in only one of these subjects. The highest risk HLA genotype $D R B 1 * 03-D Q A 1 * 0501$ $D Q B 1 * 0201 / D R B 1 * 04-D Q A 1 * 0301-D Q B 1 * 0302$ was present in $6 \%$ of the 210 unaffected grandparents who had provided a genetic sample, $65 \%$ had either one or both of the above haplotypes and $15 \%$ had the protective HLA haplotype $D Q B 1 * 0602$.

Transmission of HLA class II haplotypes. Eighty-three grandparents (from 79 families) were assessed for HLA DRB1-DQA1-DQB1 haplotype transmission. Families where both grandparents had diabetes and those with uninformative transmission were excluded from the analysis. Parents transmitted the haplotype they had received from the affected grandparent to their affected child in 59\% of the families rather than $50 \%$ as expected $(p=0.02)$. Transmission was analysed according to the treatment of the affected grandparent: insulin treated $(n=26)$, tablet treated $(n=40)$ and diet alone $(n=17)$. Parents transmitted the haplotype from the affected grandparent in $65 \%$ of the insulin group $(p=0.027), 60 \%$ of the tablet group $(p=0.07)$ and $47 \%$ of the diet treated group $(p=0.7)$. 


\section{Discussion}

We found that $7.3 \%$ of grandparents of children with Type 1 diabetes also had a diagnosis of diabetes. This estimate should be viewed with some caution. One potential source of bias lies in the fact that many of the grandparents had either died or were unavailable for study. The subgroup tested did however resemble the larger group of subjects who could not be studied. A second concern is that many reportedly non-diabetic grandparents are likely to have undiagnosed diabetes, since this is common in this age group [11, 12]. We attempted to evaluate the extent of this problem by measuring $\mathrm{HbA}_{1 \mathrm{c}}$ in the control group of unaffected grandparents recruited for genetic analysis. The biological variance of $\mathrm{HbA}_{1 \mathrm{c}}$ is however such that it has limited value as a screening test for diabetes [13], and we encountered a high proportion of moderately increased values which were difficult to evaluate. The estimates we have used therefore exclude previously unrecognised diabetes, and under-represent the true prevalence of diabetes in the grandparental generation. However epidemiological surveys in United Kingdom populations found a somewhat lower prevalence of known diabetes (4.6-5.3\%) across a similar age group $[14,15]$.

Using the classification scheme outlined above, $2.2 \%$ had features of Type 1 diabetes. Our classification scheme was based upon early requirement for insulin, defined as insulin treatment within 12 months of diagnosis, or islet autoantibodies. Childhood onset Type 1 diabetes typically presents with the full range of islet autoantibodies [7, 16, 17, 18], but ICA and GAD antibodies are characteristic of late onset immune-mediated diabetes [19]. ICA diminish over time but GAD antibodies persist for many years after diagnosis $[20,21,22]$. We used the presence of these autoantibodies to identify immune-mediated diabetes in grandparents who did not require insulin or had a delayed requirement for it. HLA haplotypes conferring increased susceptibility to Type 1 diabetes can be used to support this diagnosis in other populations, but there will inevitably be an ascertainment bias in the predecessors of affected children. The presence of high risk haplotypes in affected grandparents is therefore difficult to interpret and we have excluded this criterion from our classification scheme. However the observation that $66 \%$ had a high risk haplotype implies that a $2.2 \%$ prevalence of immune-mediated diabetes could well be an underestimate in this generation. Since population studies of late onset diabetes have typically allocated diabetes type on the basis of treatment rather than measurement of immune markers, we have no comparable estimate of the rate of immune-mediated diabetes in the background population.

We went on to look at transmission of HLA haplotypes from affected grandparents to the proband. A parent receives one HLA haplotype from each grandparent, and has a 50\% chance of transmitting each haplotype to his or her offspring. We found, however, that parents transmitted the haplotype from the affected grandparent to the affected grandchild more often than expected $(p=0.02)$. When the grandparents were sub-divided according to treatment group, the transmission rate of HLA haplotypes from affected grandparents was different from that expected in the insulin group $(65 \%, p=0.027)$ and approached significance in the tablet-treated group $(60 \% p=0.07)$ but was at the expected rate in the diet-treated group (47\% $p=0.7)$. This is the only study to have assessed transmission of Type 1 diabetes across three generations, and even in this large family study the number of available and informative families remains below 100. Despite this limitation, the increased frequency of haplotype transmission between affected generations suggests that HLA class II susceptibility is shared between grandparents with diabetes and their affected grandchildren.

The clinical and immune features of Type 1 diabetes are therefore over-represented in grandparents of affected children, as compared with the general population. Although the grandchildren presented with classic Type 1 diabetes, its characteristic features were often absent in grandparents, who presented later in life and did not necessarily have an early requirement for insulin. Evidence of increased haplotype sharing strengthens the suggestion that grandparent and grandchild are affected by the same disease process, despite the difference in presentation.

In conclusion, this study has confirmed that treatment-based classification will underestimate the frequency of autoimmune diabetes in family members. It suggests that autoimmune diabetes is over-represented in grandparents of children with Type 1 diabetes, as compared with the general population, and shows that they are more likely to transmit HLA haplotypes to their affected grandchildren. These observations would be consistent with the suggestion that the increasing incidence of childhood onset autoimmune diabetes in the second half of the 20th century represents earlier manifestation of a condition that affected older subjects in previous generations [23]. If so, this would help to explain the persistence of an otherwise lethal trait.

Acknowledgements. This study was supported by Diabetes UK. We acknowledge the continuing help and support of the children and their families; of the study fieldworkers; and of paediatricians, physicians, and diabetes nurse specialists in the Oxford region. We also wish to thank the project administrators, Isabel Wilson and Suzanne Weeks and the BOX study fieldworkers for all their help. We are grateful to A. Williams and A. Norcross for their technical assistance. 


\section{Reference}

1. Alberti KGMM, Zimmet PZ (1998) Definition, diagnosis and classification of diabetes mellitus and its complications part 1: diagnosis and classification of diabetes mellitus. Provisional report of a WHO consultation. Diabet Med 15:539-553

2. Douek IF, Gillespie KM, Bingley PJ, Gale EAM (2002) Diabetes in the parents of children with type 1 diabetes. Diabetologia 45:495-501

3. Onkamo P, Väänänen S, Karvonen M, Tuomilehto J (1999) Worldwide increase in incidence of Type 1 diabetes - the analysis of the data on published incidence trends. Diabetologia 42:1395-1403

4. EURODIAB ACE Study Group (2000) Variation and trends in incidence of childhood diabetes in europe. Lancet 355:873-876

5. Fagot-Campagna A, Narayan KMV, Imperatore G (2001) Type 2 diabetes in children. BMJ 322:377-378

6. Bingley PJ, Gale EAM (1989) Incidence of insulin dependent diabetes in England: a study in the Oxford region, 1985-1986. BMJ 298:558-560

7. Bingley PJ, Bonifacio E, Williams AJK, Genovese S, Bottazzo GF, Gale EAM (1997) Prediction of IDDM in the General Population. Strategies based on combinations of autoantibody markers. Diabetes 46:1701-1710

8. Verge CF, Stenger D, Bonifacio E et al. (1998) Combined use of autoantibodies (IA-2 autoantibody, GAD autoantibody, insulin autoantibody, cytoplasmic islet cell antibodies) in type 1 diabetes. Combinatorial islet autoantibody workshop. Diabetes 47:1857-1866

9. Gillespie KM, Valovin SJ, Saunby J et al. (2000) HLA class II typing of whole genome amplified mouth swab DNA. Tissue Antigens 56:530-538

10. Bunce M, O’Neill CM, Barnado MCNM, Krausa P, Morris PJ, Welsh KI (1995) Phototyping: comprehensive DNA typing for HLA-A, B, C, DRB1, DRB3, DRB4, DRB5 and DQB 1 by PCR with 144 primer mixes utilising sequencespecific primers (PCR-SSP). Tissue Antigens 46:355367

11. Lawrence JM, Bennett P, Young A, Robinson AM (2001) Screening for diabetes in general practice: cross sectional population study. BMJ 323:548-551
12. Croxson SC, Burden AC, Bodington M, Botha JL (1991) The prevalence of diabetes in elderly people. Diabet Med 8:28-31

13. Kilpatrick ES, Maylor PW, Keevil BG (1998) Biological variance of glycated hemoglobin. Diabetes Care 21:261-264

14. Gatling W, Budd S, Walters D, Mullee MA, Goddard JR, Hill RD (1998) Evidence of an increasing prevalence of diagnosed diabetes mellitus in the Poole area from 1983 to 1996. Diabet Med 15:1015-1021

15. Morris AD, Boyle DIR, MacAlpine R et al. (1997) The diabetes audit and research in Tayside Scotland (DARTS) study: electronic record linkage to create a diabetes register. BMJ 315:524-528

16. Verge CF, Howard NJ, Rowley MJ et al. (1994) Anti-glutamate decarboxylase and other antibodies at the onset of childhood IDDM: a population based study. Diabetologia 37:1113-1120

17. Vandewalle CL, Falorni A, Svanholm S et al. (1995) High diagnostic sensitivity of glutamate decarboxylase autoantibodies in insulin-dependent diabetes mellitus with clinical onset between age 20 and 40 years. J Clin Endocrinol Metab 80:846-851

18. Gorus FK, Goubert P, Semakula C et al. (1997) IA-2-autoantibodies complement $\mathrm{GAD}_{65}$-autoantibodies in newonset IDDM patients and help predict impending diabetes in their siblings. Diabetologia 40:95-99

19. Li H, Isomaa B, Taskinen MR, Groop L, Tuomi T (2000) Consequences of a family history of type 1 and type 2 diabetes on the phenotype of patients with type 2 diabetes. Diabetes Care 23:589-594

20. Savola K, Sabbah E, Kulmala P, Vähäsalo P, Ilonen J, Knip M (1998) Autoantibodies associated with type 1 diabetes mellitus persist after diagnosis in children. Diabetologia 41:1293-1297

21. Decochez K, Tits J, Coolens JL et al. (2000) High frequency of persisting or increasing islet-specific autoantibody levels after diagnosis of type 1 diabetes presenting before 40 years of age. Diabetes Care 23:838-844

22. Dromey JA, Mijovic CH, Christie MR et al. (2000) HLA linked persistence of antibodies to GAD 65 in patients with 50 years of type 1 diabetes. Diabet Med 17: A73

23. Gale EAM (2002) The rise of childhood Type 1 diabetes in the twentieth century. Diabetes 51:3353-3361 